\title{
Tratamiento estético de defecto de esmalte utilizando tratamiento combinado con resina infiltrante: Reporte de caso.
}

\section{Aesthetic treatment of enamel defect using combined therapy with resin infiltration: Case report}

\author{
Catalina Torres-Rabello ${ }^{1}$, Catalina Rodríguez-Alvarez ${ }^{2,3}$, Paola Barnafi-Retamal ${ }^{3}$, Camila Corral-Núñez ${ }^{3^{*}}$.
}

1. Facultad de Odontología, Universidad de Chile, Santiago, Chile.

2. Dentista en Etapa de Destinación y Formación, Consultorio General Rural Pozo Almonte, Chile. 3. Departamento de Odontología Restauradora, Facultad de Odontología, Universidad de Chile, Santiago, Chile.

* Correspondencia Autor: Camila Corral Núñez | Dirección: Olivos 943, Independencia, Santiago, Chile. | Teléfono: +562 29791742 | E-mail: camila. corral@odontologia.uchile.cl

Trabajo recibido el 30/03/2020.

Trabajo revisado 15/06/2020

Aprobado para su publicación el 03/08/2020

\begin{abstract}
RESUMEN
La presencia de defectos en el esmalte puede afectar negativamente la autoestima de pacientes jóvenes, haciendo muchas veces necesario realizar un tratamiento estético. El tratamiento con resinas infiltrantes, permite obtener resultados estéticos sin realizar remoción del tejido dentario. Sin embargo, en casos de defectos de esmalte con fracturas post-eruptivas, la resina infiltrante por sí sola, no permite obtener un resultado óptimo. El presente reporte utiliza la combinación de resina infiltrante con resina compuesta directa para obtener resultados estéticos. De esta manera, al infiltrar primero, se mejora el aspecto estético de la lesión y también las características adhesivas del esmalte defectuoso, para posteriormente restaurar el contorno perdido aplicando una delgada capa de resina compuesta de translucidez media.
\end{abstract}

PALABRAS CLAVE:

Resina infiltrante; Hipomineralización incisivo molar; Tratamiento mínimamente invasivo.

Int. J. Inter. Dent Vol. 14(2); 177-180, 2021.

\section{ABSTRACT}

The presence of enamel defects can affect negatively the self-esteem of young patients, making it necessary to carry out an aesthetic treatment. Resin infiltration treatment provides aesthetic results without the necessity of removing the defective dental tissue. However, in cases of enamel defects with post-eruptive fractures, the treatment with resin infiltration by itself does not achieve optimal results. This report uses the combination of resin infiltration with direct resin composite to obtain an aesthetic result. This way, by first infiltrating, the aesthetic appearance of the lesion is improved, as well as the adhesive characteristics of the enamel, and subsequently the contour is restored by applying a thin layer of medium translucency resin composite.

KEY WORDS:

Resin infiltration; Molar incisor hypomineralization; Minimal invasive treatment.

Int. J. Inter. Dent Vol. 14(2); 177-180, 2021.

\section{INTRODUCCIÓN}

Durante la formación del esmalte pueden ocurrir diversos problemas que originan defectos en el espesor y/o en la mineralización de este tejido(1). La hipomineralización incisivo molar (HIM) es una patología de origen sistémico, de carácter cualitativo, que afecta el esmalte de por lo menos uno de los primeros molares permanentes, pudiendo o no incluir a los incisivos permanentes ${ }^{(2)}$. La HIM es una condición bastante frecuente en la población; se ha estimado una prevalencia global de $13.1 \%$, con un $27.4 \%$ de los casos en los que se considera necesitan tratamiento debido a dolor, hipersensibilidad o fracturas posteruptivas ${ }^{(3)}$. Esta patología se ha descrito con una gran variabilidad clínica, pudiendo ir desde simples opacidades hasta la ausencia de molares por extracciones, incluyendo restauraciones atípicas y fracturas post-eruptivas ${ }^{(4)}$. Las fracturas posteruptivas ocurren en zonas de esmalte con opacidades, las cuales debido a su mayor porosidad son más débiles, por tanto, presentan mayor facilidad para fracturarse ${ }^{(1,5)}$.

Se ha observado que la presencia de defectos de esmalte puede afectar negativamente la calidad de vida o autoestima de niños ${ }^{(6)}$. Es por esto que se han detallado diferentes alternativas de tratamiento para resolver este problema estético. Dentro de ellas se han descrito el uso de prótesis fija unitaria ${ }^{(7)}$, la remoción del defecto utilizando fresas ${ }^{(8)}$ o microabrasión ${ }^{(9)}$ y su posterior restauración con resina compuesta. Sin embargo, estas opciones conllevan la remoción irreversible de tejido dentario, con el consiguiente ingreso al ciclo de restauración. La adhesión directa a esmalte afectado por hipomineralización es difícil, debido a las alteraciones físicas y químicas que este presenta ${ }^{(10)}$.Se ha planteado que el factor limitante es la marcada porosidad que presenta este esmalte hipomineralizado y por ello algunos autores han recomendado extender los márgenes de la preparación a esmalte sano(11). Una reciente revisión sistemática enfatiza la necesidad de mejorar la adhesión a esmalte afectado por $\mathrm{MIH}$, estableciendo que, la evidencia disponible actualmente es muy limitada para resolver el problema de la adhesión a este tejido(12). Diferentes estrategias se han estudiado, como la desproteinización previa del esmalte, el uso de distintos sistemas adhesivos y el uso de resinas infiltrantes ${ }^{(11,12)}$. Sin embargo, actualmente debido a la poca cantidad de estudios clínicos, no es posible dar recomendaciones claras sobre técnica a utilizar ${ }^{(10,12,13)}$.

Las resinas infiltrantes se han utilizados para estos defectos por 
hipomineralización de esmalte, las cuales permiten enmascarar el cambio de coloración ${ }^{(14)}$. Sin embargo, cuando el esmalte también está afectado en su grosor, las resinas infiltrantes no permiten restaurar la anatomía del esmalte defectuoso, por tanto, es necesario el uso combinado con otro material, como las resinas compuestas. En el presente reporte se relata el caso de un paciente afectado por HIM en un incisivo central superior, con mancha blanca/amarilla y fractura post-eruptiva la cual fue tratada con resina infiltrante y resina compuesta directa como medida mínimamente invasiva, solucionando el problema estético.

\section{INFORME DEL CASO}

Paciente sexo masculino de 16 años de edad, se presenta a la Clínica Odontológica del Niño y Adolescente, Facultad de Odontología, Universidad de Chile. Su motivo de consulta es su disconformidad con la manera que se ve uno de sus dientes anteriores. Paciente sistémicamente sano, antecedentes de anamnesis y examen intraoral se detallan en Tabla 1. Al examen específico de 2.1 se observa defecto de esmalte, en tercio medio/incisal de superficie vestibular, con fractura post-eruptiva y defecto de mancha blanca/amarilla (Figura 1), de acuerdo a clasificación de severidad de HIM de Mathu-Muju y Wright se considera como moderada por presentar ruptura limitada a una superficie sin sensibilidad ${ }^{(15)}$. Se le explicaron las opciones de tratamiento al adolescente y su madre, los cuales dieron su consentimiento para realizar tratamiento odontológico integral del paciente, incluyendo en la fase restauradora el tratamiento de infiltración y posterior restauración de lesión en 2.1. Se realizó consejería de dieta y de higiene oral de manera de controlar la gingivitis y enfermedad de caries, previo a comenzar la fase restauradora.
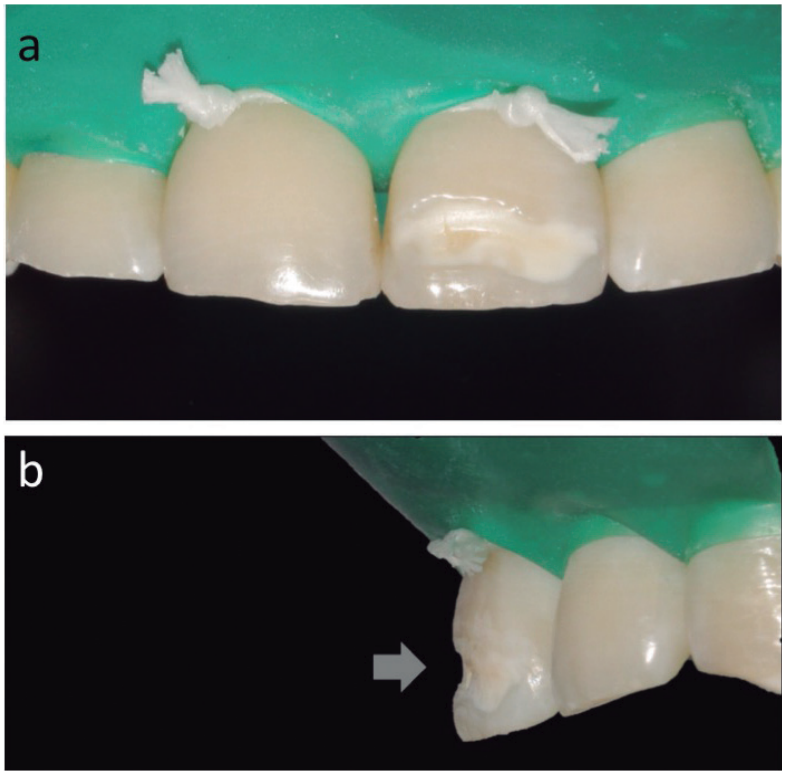

Figura 1. Fotografía clínica de defecto de esmalte en 2.1 de mancha blanca y amarilla por vestibular (a), en imagen lateral se observa defecto en grosor del esmalte (b).

Se limpió la superficie del diente 2.1 con escobilla suave y se aislaron de manera absoluta los dientes del sector anterosuperior (Figura 2). La mancha fue grabada con gel de ácido clorhídrico al 15\% (ICON-etch; DMG, Hamburgo, Alemania) por 2 minutos, abarcando la zona de la lesión y al menos $1 \mathrm{~mm}$ alrededor de ella, esta zona fue luego lavada por 30 segundos. Se aplicó posteriormente una solución de etanol, presente en formato de jeringa en el kit de Icon (Icon-Dry DMG, Hamburgo, Alemania), por 30 segundos para secar la lesión y observar el cambio estético de la mancha. Este procedimiento se repitió dos veces más. Luego se realizó la infiltración con Icon-Infiltrant utilizando el aplicador para lesiones de superficies libres. Siguiendo las instrucciones del fabricante, se aplicó una cantidad generosa de la resina infiltrante, realizando movimientos circulares con el aplicador de manera de facilitar la infiltración. Esto se realizó por 3 minutos. Luego, se removieron los excesos de material con sonda y seda dental y se fotopolimerizó por 40 segundos. E procedimiento de infiltración se repitió, aplicando Icon-Infiltrant por 3 minutos, removiendo excesos y fotopolimerizando nuevamente. Luego de la infiltración se observa cambio de color de la lesión, camuflándose con el color del diente, sin embargo, esta aún es distinguible.
Tabla 1: Antecedentes de anamnesis y examen intraoral del paciente.

\begin{tabular}{|c|c|c|c|c|}
\hline \multirow{3}{*}{$\begin{array}{l}\text { Antecedentes } \\
\text { materno- } \\
\text { infantiles }\end{array}$} & \multirow{3}{*}{\multicolumn{4}{|c|}{$\begin{array}{l}\text { Embarazo normal con parto vaginal, de término, } \\
\text { pero inducido. } \\
\text { Nacimiento: coroamnionitis (estuvo } 2 \text { días en incu- } \\
\text { badora). } \\
\text { Lactancia: materna y artificial desde el nacimiento. }\end{array}$}} \\
\hline & & & & \\
\hline & & & & \\
\hline & \multirow{2}{*}{\multicolumn{4}{|c|}{$\begin{array}{l}\text { Actualmente, sin patologías médicas. } \\
\text { Asmático entre } 6 / 7-12 \text { años. En tratamiento con } \\
\text { budesonida, loratadina y salbutamol SOS, durante } \\
\text { ese período. }\end{array}$}} \\
\hline $\begin{array}{l}\text { Antecedentes } \\
\text { médicos }\end{array}$ & & & & \\
\hline \multirow[t]{2}{*}{ Hábitos } & \multirow{2}{*}{\multicolumn{4}{|c|}{$\begin{array}{l}\text { No relata consumo de alcohol, tabaco ni drogas. } \\
\text { Tiene } 6 \text { momentos de ingesta de comida al día, de } \\
\text { los cuales } 4 \text { son con carbohidratos, presenta un alto } \\
\text { consumo de azúcar en bebidas. } \\
\text { Cepillado 1-2 veces al día con pasta dental de } 1.450 \\
\text { ppm de flúor. }\end{array}$}} \\
\hline & & & & \\
\hline \multirow{4}{*}{$\begin{array}{l}\text { Examen } \\
\text { intraoral }\end{array}$} & \multirow{2}{*}{ Examen Periodontal Básico } & 0 & 1 & 1 \\
\hline & & 1 & 2 & 1 \\
\hline & \multicolumn{4}{|c|}{$\begin{array}{l}\text { Índice gingival: 0,17, inflamación gingival leve. } \\
\text { Índice de placa: } 52,6 \%\end{array}$} \\
\hline & \multicolumn{4}{|c|}{$\begin{array}{l}\text { Hipomineralizaciones de esmalte presentes en } 1.6 \\
\text { (asociada a lesión de caries ICDAS } 3 \text { ), 2.1, 2.6, 3.6. }\end{array}$} \\
\hline
\end{tabular}

Posterior a ello se restauró con Filtek XT Z350 A2 Body (3M ESPE, Saint Paul, MN, USA, Figura 3), directamente sobre la lesión infiltrada y se pulió con discos Sof-Lex (3M ESPE, Saint Paul, MN, USA). Se observa el cambio en el contorno del diente, restableciendo la anatomía del diente en su superficie vestibular, y cambiando la apariencia de la mancha del diente. El paciente fue citado a control para realizar pulido final. Tanto el paciente como su madre quedaron conformes con el resultado.

\section{DISCUSIÓN}

La mayoría de los defectos de esmalte son aceptados por el paciente y no generan un rechazo(16). Sin embargo, si estos defectos de esmalte son más severos y causan molestias en el paciente, se puede considerar realizar tratamientos que logren mejorar la estética del diente afectado. La corrección de estos defectos con procedimientos convencionales, puede requerir la eliminación de tejido sano debido a la preparación del diente. Sin embargo, esto debe ser evitado en lo posible.

En los últimos años, han aparecido las resinas infiltrantes, las cuales se utilizan principalmente en el tratamiento de lesiones incipientes de caries, de tipo mancha blanca no cavitada. Sin embargo, su uso se ha extendido para enmascarar algunas alteraciones del desarrollo del esmalte, como en casos de fluorosis e hipomineralizaciones. Un aspecto positivo de la técnica de infiltración es que cambia las propiedades ópticas del esmalte hipomineralizado ${ }^{(17)}$. El índice de refracción de la resina infiltrante es más cercano al de la hidroxiapatita que al del agua o aire; de esta forma, cuando el material infiltra las porosidades se genera un efecto enmascarador, que lo hace más cercano a la apariencia del esmalte sano ${ }^{(18)}$. La resina infiltrante, puede rellenar la lesión generando esta mejoría estética del defecto de esmalte, especialmente en alteraciones leves a moderadas; sin embargo, en casos más severos, el enmascaramiento de color no siempre es óptimo. Además, como se muestra en el presente reporte, la técnica de infiltración de resina no es capaz de rellenar el defecto cuando existen también defectos en el grosor, por ello se propone la combinación de la infiltración con la técnica restaurativa convencional usando resinas compuestas para restaurar el contorno y anatomía defectuosa.

Esta combinación podría considerarse como microinvasiva, ya que solo se remueve una capa muy superficial de esmalte (en el rango micrométrico) producto de la acción del ácido clorhídrico al $15 \%{ }^{(19)}$. La remoción de esta capa más superficial de esmalte es vital para una exitosa infiltración, ya que esta se genera principalmente a través de capilaridad $^{(19)}$. De esta forma se modifica la composición del esmalte defectuoso y mejora su aspecto estético, permitiendo el uso de grosores menores de resina compuesta y con algún grado de translucidez, para resolver el problema estético y recuperar la anatomía del diente.

Actualmente, existe evidencia de que el esmalte afectado por HIM 

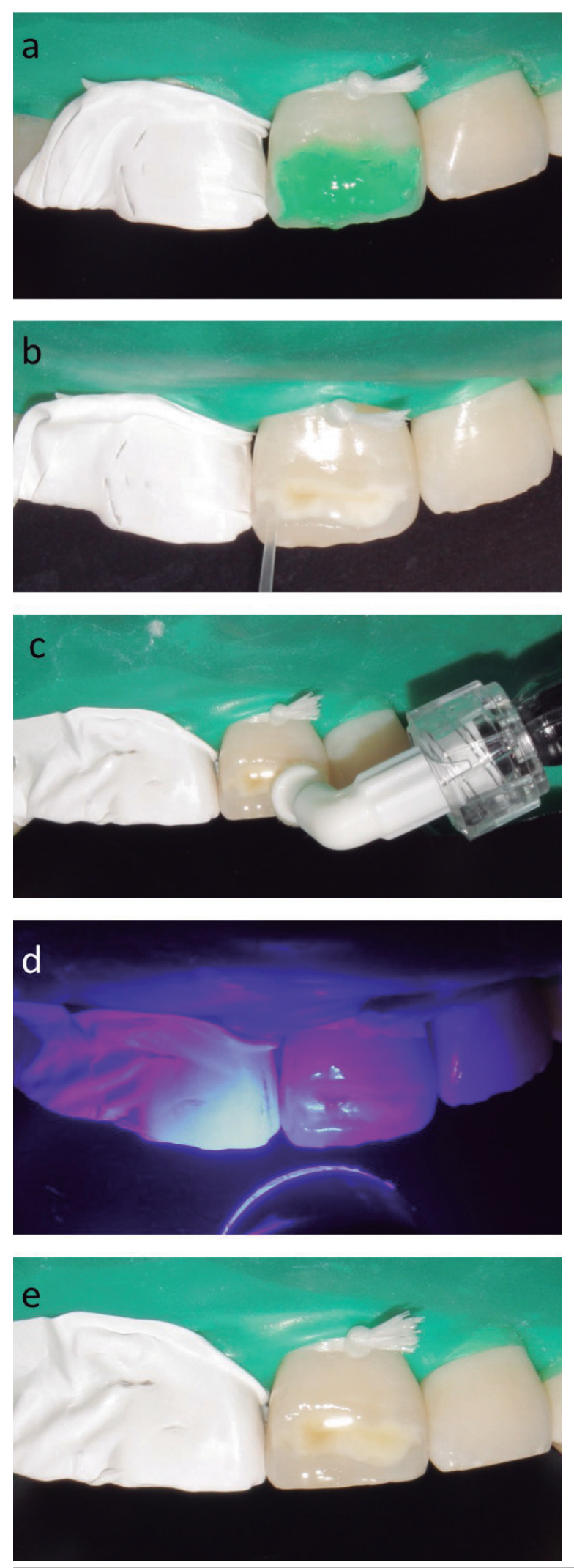

Figura 2. Secuencia clínica de procedimiento de infiltración del defecto de esmalte. Grabado de la lesión con ácido clorhídrico (a), secado con solución de etanol (b), infiltración (c), fotopolimerización (d), post-infiltración (e).
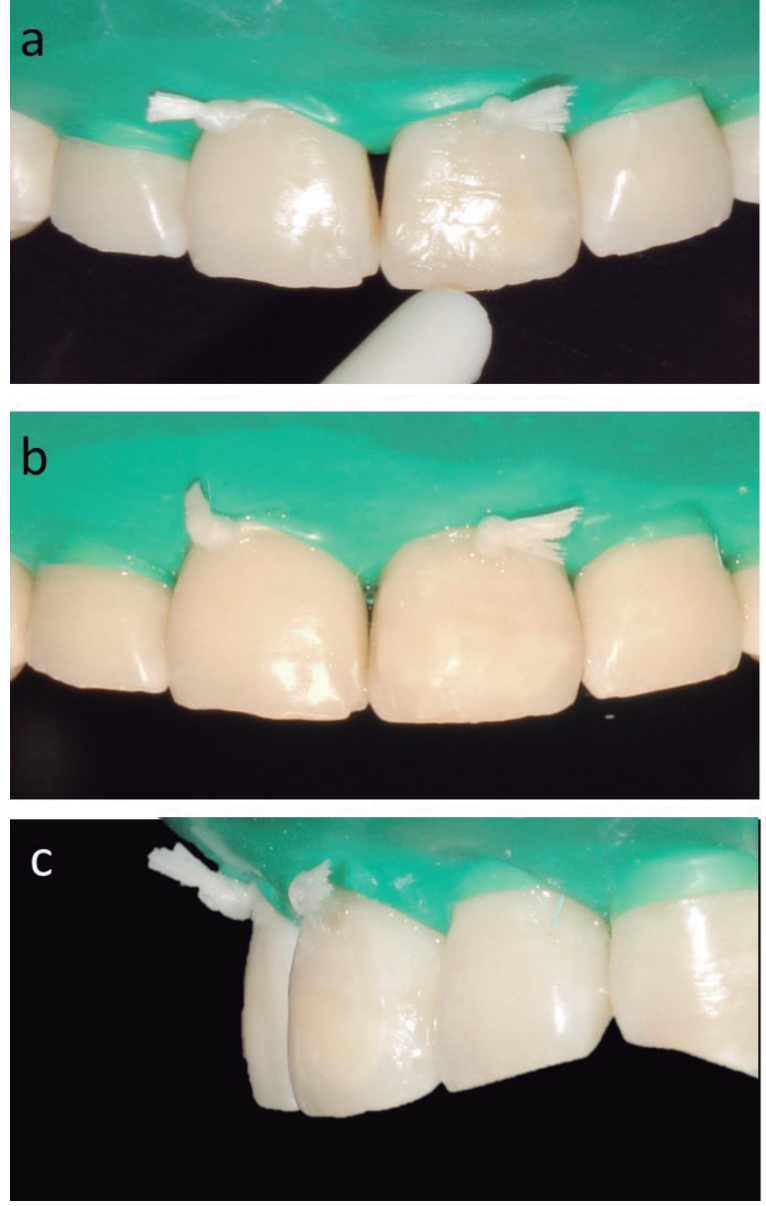

Figura 3. Secuencia clínica de restauración directa con resina compuesta.

presenta características diferentes a la del esmalte normal, con reducción en cantidad y calidad mineral, menor dureza y módulo de elasticidad, entre otros ${ }^{(10)}$. De manera relevante para estos casos, presenta también una porosidad aumentada, por lo que se han sugerido modificaciones a las estrategias adhesivas convencionales ${ }^{(10)}$. Si bien, actualmente existen sistemas adhesivos y de resinas compuestas, que permiten obtener excelentes resultados estético(20), existe evidencia, de que la porosidad del esmalte afectado por HIM es el factor limitante para la adhesión con sistemas adhesivos convencionales ${ }^{(11)}$ y que la infiltración previa con resina infiltrante genera un mejor sustrato para la posterior adhesión de la resina compuesta convencional(21). Además, también se ha establecido que el grabado con ácido fosfórico del esmalte con HIM genera patrones de grabado deficientes y anormales, con más cracks y poros ${ }^{(22)}$. La infiltración de estas lesiones con resina infiltrante podría por un lado reducir la permeabilidad del esmalte, y con ello la hipersensibilidad que se expresa en algunos casos, y por otro aumentar la dureza y resistencia a la flexión del esmalte defectuoso, optimizando también la capacidad de adhesión a esta superficie tratada, mejorando probablemente la longevidad de las restauraciones ${ }^{(10,19,21)}$.

El manejo clínico de HIM es complejo y se ha asociado a una alta necesidad de tratamiento. Niños afectados con HIM tienen una tasa mayor de fracaso de sus restauraciones y requieren más re-tratamientos que niños sin $\mathrm{HIM}^{(23,24)}$. Por tanto, han surgido diferentes investigaciones y estrategias para intentar mejorar la adhesión de resina a dientes afectados por $\mathrm{MIH}$, dentro de ellas está el uso de infiltrantes ${ }^{(11-13,25)}$. Lamentablemente, actualmente no hay consenso sobre técnica adhesiva a utilizar en casos de incisivos afectados por $\mathrm{HIM}^{(12)}$. Revisiones han establecido, que con la evidencia disponible no es posible dar recomendaciones precisas a clínicos para el tratamiento de incisivos afectados y se sugiere realizar mayor cantidad de estudios clínicos, con mayor período de seguimiento ${ }^{(12,13,26)}$. Sin embargo, hay claridad en que en estos casos es importante considerar intervalos cortos entre citaciones de revisión, monitoreo y reevaluación, además de optar por terapias de reparación y reacondicionamiento en caso de que las restauraciones presenten defectos ${ }^{(27)}$. En el caso presentado es relevante para el pronóstico del tratamiento disminuir y mantener controlado el riesgo de enfermedad periodontal y de caries, más aun considerando que se ha 
establecido una asociación entre HIM y caries dental ${ }^{(27)}$. El tratamiento incluyó un fuerte componente de manejo de factores de riesgo con enfoque centrado en el paciente. Se realizó consejería de manera que el paciente incorpore a su rutina diaria el cepillado eficiente, dos veces al día, con pasta $\geq 1450 \mathrm{ppm}$ de flúor, consejería de dieta y se planificaron aplicaciones de barniz de flúor cuatro veces al año (paciente vive en zona de agua fluorada), con intervalos de citación cada 3 meses, siguiendo las recomendaciones para manejo de caries en condiciones de alto riesgo cariogénico ${ }^{(28)}$.

La técnica combinada de infiltración y posterior restauración es una técnica fácil, conservadora y mínimamente invasiva, especialmente en pacientes jóvenes. Este reporte de caso muestra la resolución de un problema estético sin eliminar tejidos dentarios, que, aunque presentan defectos en su grado de mineralización se encuentran sanos.

\section{CONCLUSIÓN}

Es posible realizar tratamiento restaurador estético, de defecto de esmalte por hipomineralización incisivo molar, sin realizar remoción de tejido dentario, utilizando una combinación de resina infiltrante y resina compuesta directa. Sin embargo, con la evidencia disponible no es posible dar una recomendación precisa al clínico para el tratamiento de incisivos afectados por HIM con fractura post-eruptiva ya que se requieren mayor cantidad de estudios clínicos, con mayor período de seguimiento para ello.

\section{RELEVANCIA CLÍNICA}

El caso reportado demuestra que es posible obtener resultado estético en defectos de esmalte severos utilizando solo terapias microinvasivas, que permiten preservar los tejidos dentarios afectados.

\section{CONFLICTO DE INTERÉS}

Los autores declaran no presentar conflicto de interés

\section{Bibliografía}

1. Garg N, Jain AK, Saha S, Singh J. Essentiality of early diagnosis of molar incisor hypomineralization in children and review of its clinical presentation, etiology and management. Int J Clin Pediatr Dent. 2012;5(3):190-6.

2. Koch G, Hallonsten AL, Ludvigsson N, Hansson BO, Holst A, Ullbro C. Epidemiologic study of idiopathic enamel hypomineralization in permanent teeth of Swedish children. Community Dent Oral Epidemiol. 1987;15(5):279-85.

3. Schwendicke F, Elhennawy K, Reda S, Bekes K, Manton DJ, Krois J. Global burden of molar incisor hypomineralization. J Dent. 2018;68(2018):10-8.

4. Lygidakis NA, Wong F, Jalevik B, Vierrou AM, Alaluusua S, Espelid I. Best Clinical Practice Guidance for clinicians dealing with children presenting with Molar-IncisorHypomineralisation (MIH): An EAPD Policy Document. Eur Arch Paediatr Dent. 2010;11(2):75-81.

5. Weerheijm KL, Duggal M, Mejare I, Papagiannoulis L, Koch G, Martens LC, et al. Judgement criteria for molar incisor hypomineralisation $(\mathrm{MIH})$ in epidemiologic studies: a summary of the European meeting on $\mathrm{MIH}$ held in Athens, 2003. Eur $\mathrm{J}$ Paediatr Dent. 2003;4(3):110-3.

6. Gutierrez TV, Ortega CCB, Perez NP, Perez AG. impact of molar incisor hypomineralization on oral health-related quality of life in Mexican schoolchildren. $J$ Clin Pediatr Dent. 2019;43(5):324-30.

7. Lam WY, Ho EH, Pow EH. Rehabilitation of molar-incisor hypomineralization (MIH) complicated with localized tooth surface loss: a case report. Quintessence Int. 2014;45(5):377-9.

8. Carvalho LD, Bernardon JK, Bruzi G, Andrada MA, Vieira LC. Hypoplastic ename treatment in permanent anterior teeth of a child. Oper Dent. 2013;38(4):363-8.

9. Reston EG, Corba DV, Ruschel K, Tovo MF, Barbosa AN. Conservative approach for esthetic treatment of enamel hypoplasia. Oper Dent. 2011;36(3):340-3.

10. Elhennawy K, Manton DJ, Crombie F, Zaslansky P, Radlasnski RJ, JostBrinkmann $P$, et al. Structural, mechanical and chemical evaluation of molarincisor hypomineralization-affected enamel: A systematic review. Arch Oral Biol. 2017;83:272-81.

11. Krämer N, Bui Khac NN, Lücker S, Stachniss V, Frankenberger R. Bonding strategies for MIH-affected enamel and dentin. Dent Mater. 2018;34(2):331-40.

12. Lagarde M, Vennat E, Attal JP, Dursun E. Strategies to optimize bonding of adhesive materials to molar-incisor hypomineralization-affected enamel: A systematic review. Int J Paediatr Dent. 2020; 30(4):405-20

13. Borges AB, Caneppele TM, Masterson D, Maia LC. Is resin infiltration an effective esthetic treatment for enamel development defects and white spot lesions? A systematic review. J Dent. 2017:56:11-8.

14. Kim S, Kim EY, Jeong TS, Kim JW. The evaluation of resin infiltration for masking labial enamel white spot lesions. Int J Paediatr Dent. 2011;21(4):241-8.
15. Mathu-Muju K, Wright JT. Diagnosis and treatment of molar incisor hypomineralization. Compend Contin Educ Dent. 2006;27(11):604-10.

16. Wallace A, Deery C. Management of opacities in children and adolescents. Dent Update. 2015;42(10):951-4,7-8.

17. Knosel M, Eckstein A, Helms HJ. Long-term follow-up of camouflage effects following resin infiltration of post orthodontic white-spot lesions in vivo. Angle Orthod. 2019;89(1):33-9.

18. Meyer-Lueckel H, Paris S. Caries Infiltration. Caries Management- Science and clinical practice. Stuttgart: Thieme Publishing Group; 2013.

19. Meyer-Lueckel H, Paris S, Kielbassa AM. Surface layer erosion of natural caries lesions with phosphoric and hydrochloric acid gels in preparation for resin infiltration. Caries Res. 2007;41(3):223-30.

20. Cavalheiro JP, Souza MIAV, Duque CCO, Bussaneli DG, Zuanon ÂCC, Jeremias F. Esthetic rehabilitation of anterior teeth with molar-incisor hypomineralization and dental fluorosis: a case report. Gen Dent. 2020;68(3):34-9.

21. Korner P, El Gedaily M, Attin R, Wiedemeier DB, Attin T, Taubock TT. Margin integrity of conservative composite restorations after resin infiltration of demineralized enamel. J Adhes Dent. 2017;19(6):483-9.

22. Bozal C B, Kaplan A, Ortolani A, Cortese S G, Biondi A M. Ultrastructure of the surface of dental enamel with molar incisor hypomineralization $(\mathrm{MIH})$ with and without acid etching. Acta Odontol Latinoam. 2015;28(2):192-8.

23. Kotsanos N, Kaklamanos EG, Arapostathis K. Treatment management of first permanent molars in children with Molar-Incisor Hypomineralisation. Eur J Paediatr Dent. 2005;6(4):179-84.

24. Jälevik B, Klingberg GA. Dental treatment, dental fear and behaviour management problems in children with severe enamel hypomineralization of their permanent first molars. Int J Paediatr Dent. 2002;12(1):24-32.

25. Ekambaram M, Yiu CKY. Bonding to hypomineralized enamel A systematic review. Int J Adhes Adhes. 2016;69:27-32

26. Elhennawy K, Schwendicke F. Managing molar-incisor hypomineralization: A systematic review. J Dent. 2016;55:16-24.

27. Pitts N B, Ismail A I, Martignon S, Ekstrand K, Douglas G V A, Longbottom C. ICCMS ${ }^{\mathrm{TM}}$ Guide for practitioners and educators. 2014. (accessed May 2019). Available at http://doi.org/10.5281/zenodo.853106

28. Americano GC, Jacobsen PE, Soviero VM, Haubek D. A systematic review on the association between molar incisor hypomineralization and dental caries. Int $J$ Paediatr Dent. 2017;27(1):11-21. 